\title{
Subharmonic generation in Josephson junction fluxon oscillators biased on Fiske steps
}

Sørensen, Mads Peter; Christiansen, Peter Leth; Parmentier, R. D.; Skovgaard, Ove

Published in:

Applied Physics Letters

Link to article, DOI:

$10.1063 / 1.94045$

Publication date:

1983

Document Version

Publisher's PDF, also known as Version of record

Link back to DTU Orbit

Citation (APA):

Sørensen, M. P., Christiansen, P. L., Parmentier, R. D., \& Skovgaard, O. (1983). Subharmonic generation in Josephson junction fluxon oscillators biased on Fiske steps. Applied Physics Letters, 42(8), 739-741. https://doi.org/10.1063/1.94045

\section{General rights}

Copyright and moral rights for the publications made accessible in the public portal are retained by the authors and/or other copyright owners and it is a condition of accessing publications that users recognise and abide by the legal requirements associated with these rights.

- Users may download and print one copy of any publication from the public portal for the purpose of private study or research.

- You may not further distribute the material or use it for any profit-making activity or commercial gain

- You may freely distribute the URL identifying the publication in the public portal 


\title{
Subharmonic generation in Josephson junction fluxon oscillators biased on Fiske steps
}

\author{
M. P. Soerensen, P. L. Christiansen, R. D. Parmentier, a) and O. Skovgaard \\ Laboratory of Applied Mathematical Physics, The Technical University of Denmark, DK-2800 Lyngby, \\ Denmark
}

(Received 22 November 1982; accepted for publication 1 February 1983)

\begin{abstract}
Numerical integration of the perturbed sine-Gordon equation describing a long overlap-geometry Josephson junction in a magnetic field indicates a branched structure of the first Fiske step. The major portion of the step corresponds to a simply periodic fluxon oscillation whereas the branches are characterized by subharmonic generation.
\end{abstract}

PACS numbers: 74.50. $+\mathrm{r}, 85.25 .+\mathrm{k}, 84.20 . \mathrm{Pc}, 84.30 . \mathrm{Ng}$

The dynamics of fluxons on long Josephson tunnel junctions has recently attracted considerable interest. Fluxon propagation has clearly been demonstrated to be responsible for the appearance of zero-field steps $(\mathrm{ZFS})^{1-3}$ and indicated as being associated also with the appearance of Fiske steps $(\mathrm{FS})^{4-6}$ in the current-voltage $(I-V)$ characteristics of such long junctions. Moreover, associated with this propagation there is an emission of microwave radiation of very narrow linewidth from the ends of the junction, ${ }^{7}$ suggesting the possibility of interesting electronic applications.

In this letter we report on detailed numerical investigations of a perturbed sine-Gordon model of a long Josephson junction in a magnetic field. The results that have emerged give further confirmation to the fluxon propagation mechanism as being responsible also for the FS and, more importantly, show a branching of the first FS in the $I-V$ plane with the presence of subharmonic generation on the branches.

The mathematical model studied is ${ }^{3}$

$$
\begin{aligned}
& \phi_{x x}-\phi_{t z}-\sin \phi=\alpha \phi_{t}-\gamma, \\
& \phi_{x}(0, t)=\phi_{x}(L, t)=\eta .
\end{aligned}
$$

Here, $\phi$ is magnetic flux normalized to $\hbar / 2 e, x$ the longitudinal distance normalized to the Josephson penetration depth $\lambda_{J}$, and $t$ the time normalized to the inverse of the Josephson plasma frequency $\omega_{0}$. The $\gamma$ term represents a uniformly distributed bias current normalized to the maximum zero-voltage (Josephson) current $I_{0}$ appropriate to an overlap geometry. ${ }^{8}$ The term in $\alpha$ represents quasiparticle loss. The constant $\eta$ is a normalized measure of the external magnetic field which determines the boundary conditions [Eq. (1b)] at the two ends of the junction of normalized length $L$. For this study $L=5, \alpha=0.252, \eta=0.75$, and $0<\gamma<1$. These values were chosen to be similar to those used in Ref. 5. Equations ( $1 \mathrm{a})$ and $(1 \mathrm{~b})$ were integrated using the implicit finite difference method described in detail in Ref. 3, with the space and time intervals both set to 0.05 . Numerical accuracy and stability were verified by halving and doubling the space and time intervals in the computations and by carrying out the integration for long periods of time $(t \sim 1500)$ and observing that all measurable characteristics of the solutions remained stationary.

Two types of initial conditions were used: (i) a "smooth"

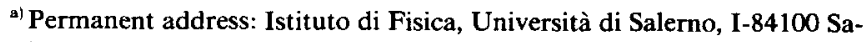
lerno, Italy.
}

initial condition, defined as

$$
\begin{aligned}
& \phi(x, 0)=F(x, 0)+G(x), \\
& \phi_{t}(x, 0)=F_{t}(x, 0),
\end{aligned}
$$

where

$$
F(x, t)=4 \tan ^{-1}\left\{\exp \left[(x-2.5+u t) /\left(1-u^{2}\right)^{1 / 2}\right]\right\},(2 \mathrm{c})
$$

in which the velocity $u(0<u<1)$ is chosen by a power balance calculation according to the value of $\gamma$, and $G(x)$ is a static solution of Eqs. (1a) and (1b) in which $\eta=0.75-F_{x}(0,0)$; (ii) a "tickled" initial condition, similar to (i) but with the further superposition of a packet of plasma oscillations, defined as

$$
H(x, t)=A \cos (k x-\omega t) \exp \left[-\left(x-x_{0}\right)^{2} / W^{2}\right],
$$

in which $k=1, \omega^{2}=1+k^{2}, 0.1 \leqslant A \leqslant 0.2,1<x_{0}<4$, and $0.1 \leqslant W \leqslant 0.2$.

The results presented refer to the situation with the junction biased on the first FS. Our most significant result is indicated in Fig. 1, which shows the $I-V$ form, on an expanded scale, of this FS. Here, and in the following, voltage is defined as $\phi_{t}$, which represents the physical voltage normalized to $\hbar \omega_{0} / 2 e$. In particular, in addition to the major portion of the step, similar to that observed by Erné et al., ${ }^{5}$ we observe two lateral branches. These branches are characterized by the generation of subharmonics in the radiation emit-

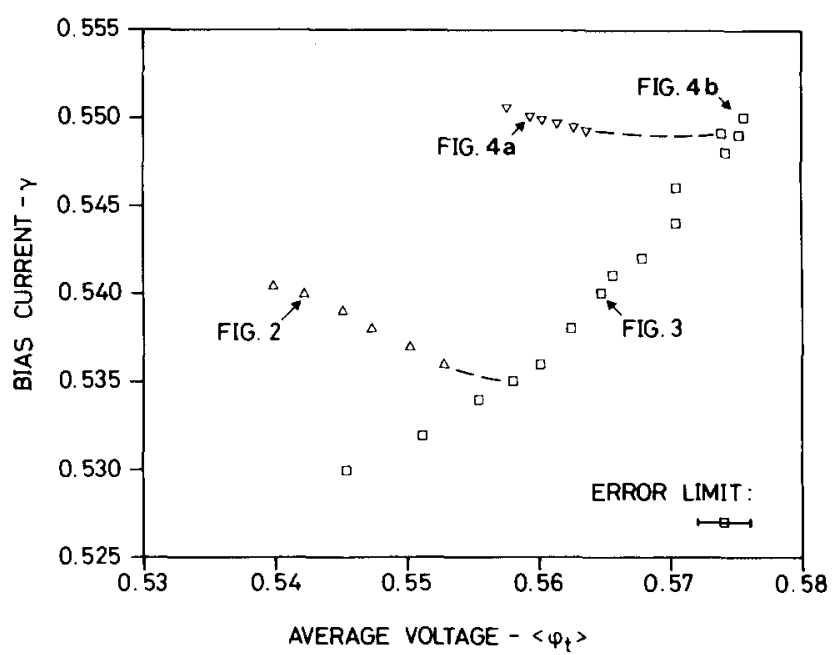

FIG. 1. Detail of current-voltage form of first Fiske step for $\eta=0.75$. Rectangles: major portion, without subharmonic generation; triangles: lateral branches, with subharmonic generation. 

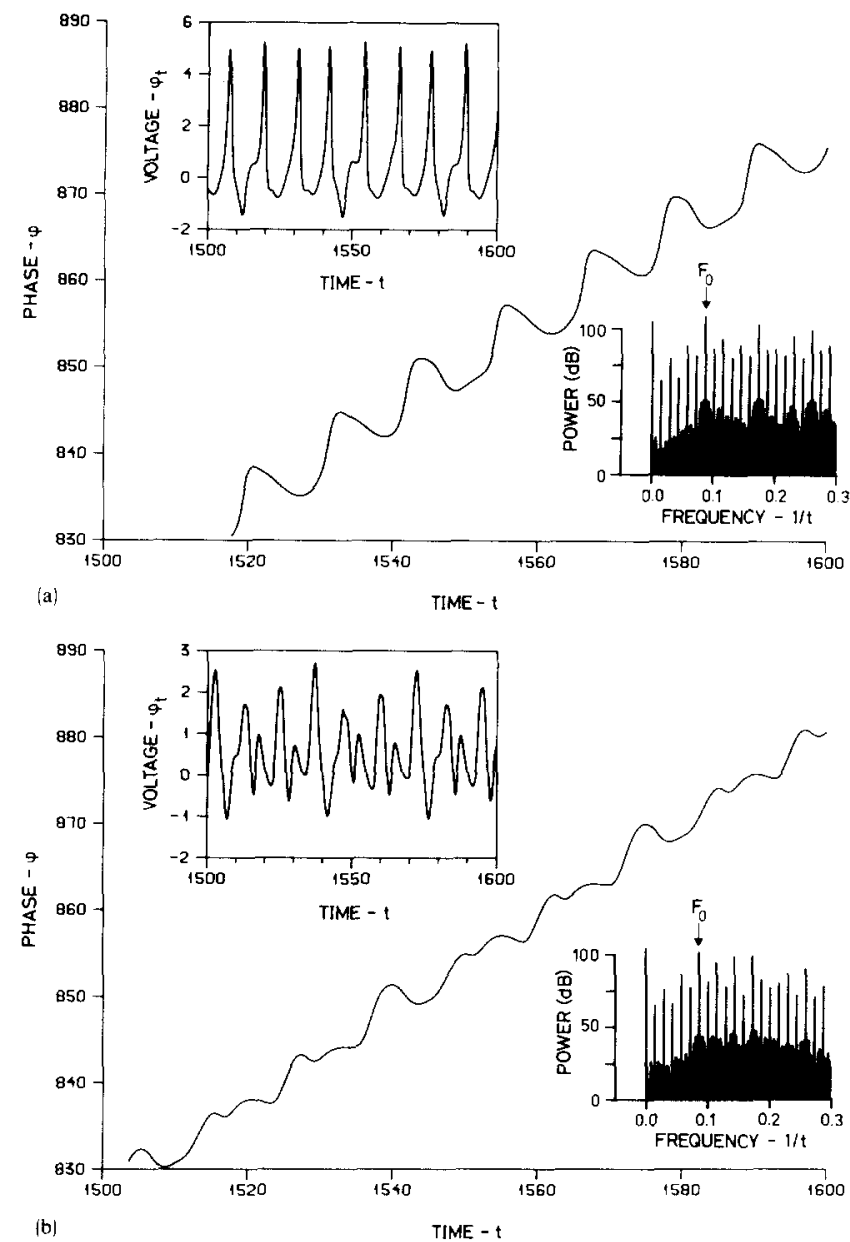

FIG. 2. Dynamics of lower subharmonic branch of first Fiske step for $\gamma=0.54, \eta=0.75$. Time evolution of phase $\phi$, voltage $\phi_{z}$ (upper inset), and power spectrum (lower inset). (a) Left junction end, (b) right junction end.

ted from the junction, whereas the major portion has no such subharmonic generation. Figures $2-4$ indicate the detailed dynamics associated with the labeled points on the $I-V$ characteristic of Fig. 1. In Figs. 2(a) and 2(b) we show the time evolution of the phase $\phi$ and the voltage $\phi_{1}$ at the two ends of the junction and the related power spectra corresponding to a point on the lower branch. The power spectra were calculated as follows: Printouts of the time evolution of $\phi_{t}$ were examined and a tentative maximum superperiod (highestorder subharmonic) established by eye. Time intervals of $\Delta t \sim 500$ of these waveforms, containing exactly an integral number of such superperiods, were then fast Fourier transformed using a simple rectangular window. For display purposes, all of the spectra have been normalized to an arbitrary value of $\phi_{t}^{2}=10^{-11}$. Solutions similar to those in Fig. 2 were invariably arrived at from "tickled" initial conditions. In this connection it is worth mentioning that the existence of the branches seems to depend only upon the fact of tickling and not upon the precise mode in which tickling is effected. This fact was established by varying the amplitude, width, and initial position of the tickling wave packet in the type (ii) initial condition and observing that the state into which the solution evolved did not change with these variations. As is apparent from Figs. 2(a) and 2(b), the fundamental period of oscillation is approximately 12 normalized time units. The
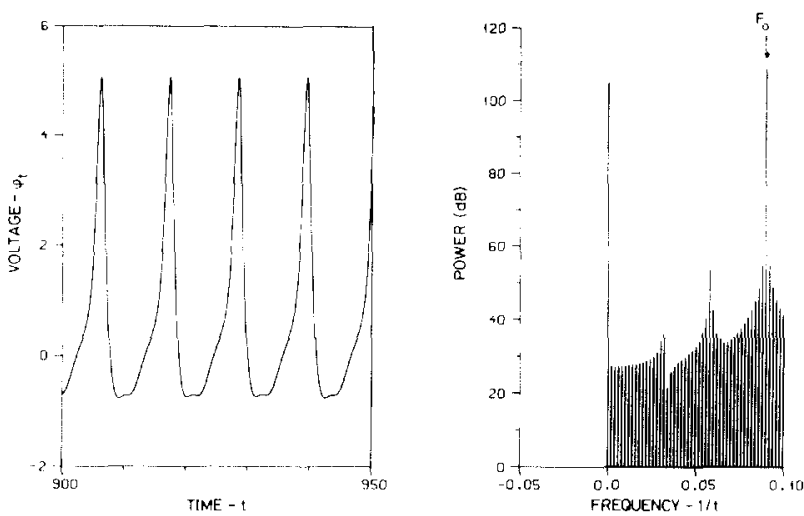

FIG. 3. Dynamics of major portion of first Fiske step for $\gamma=0.54$, $\eta=0.75$. Time evolution of voltage $\phi$, and power spectrum at left junction end.

presence of the asymmetric fluxon propagation mechanism proposed in Ref. 4 is confirmed by the phase jump of $2 \pi$ per fundamental period, ${ }^{5}$ and by the asymmetry between the time evolution at the left and right ends (note that the polarity of the magnetic field used here is opposite that used in Ref. 5, for which right and left are interchanged here with respect to that work). Particularly apparent from the insets of Fig. 2 is the strong subharmonic content of this oscillation. This is shown in detail in the power spectra of Figs. 2(a) and 2(b), which indicate the presence of third and sixth subharmonics as well as multiples of these. In contrast with the results of Fig. 2, when the "smooth" initial condition is employed with the same value of the bias, the simply periodic time evolution indicated in Fig. 3 is obtained. Although some subharmonic content is still present, it is very much reduced compared with that shown in Fig. 2.

In a similar way, higher up on the FS is a second branch of the $I-V$ curve, the power spectra of the dynamics of which are indicated in Figs. 4(a) and 4(b). In Fig. 4(a), once again, subharmonic generation is observed, this time, however, with only a dominant second subharmonic. As before, the subharmonic branch of Fig. 4(a) evolves from the type (ii) initial condition, whereas the simply periodic solution of Fig. 4(b) evolves from the type (i) initial condition.

In physical terms, the major portion of the FS corresponds to a situation in which a fluxon propagates in the field-aided direction (here, from right to left), is reflected at the end as a localized plasmon moving in the opposite direction, which in turn is reflected at the other end as a fluxon which resumes propagating exactly as before. ${ }^{4,5}$ Propagation on the subharmonic branches is almost the same, except that perfect periodicity of the overall process is resumed only after a certain number of complete back-and-forth cycles. The reason for the existence of such multiple solutions for given parameter values is not understood, but the fact is consistent with the frequently noted experimental observation of fine structure on FS. ${ }^{9}$ In the experiments, wave packets may be created by thermal fluctuations or imperfections in the junction thus giving rise to creation of subharmonics.

Of considerable interest is the manner in which the solutions evolve as the bias parameter $\gamma$ is varied in small increments during the computation. As we move along the 


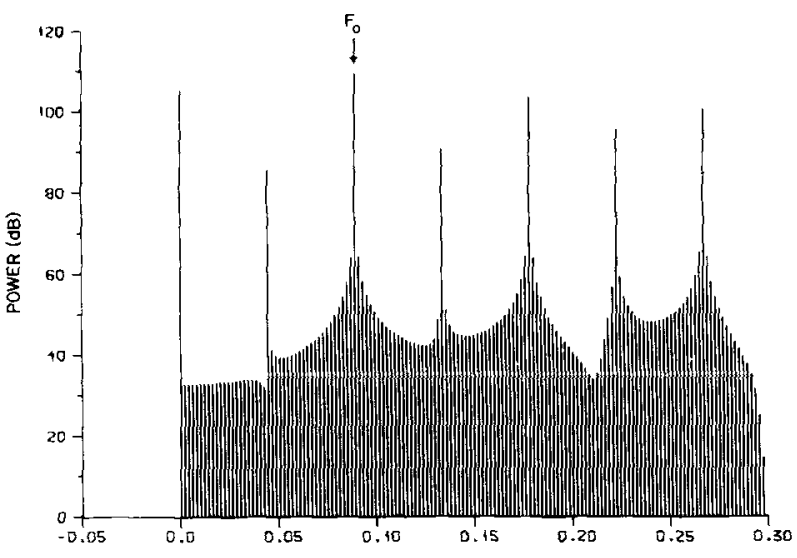

(a)

FREQUENCY $-1 / 1$

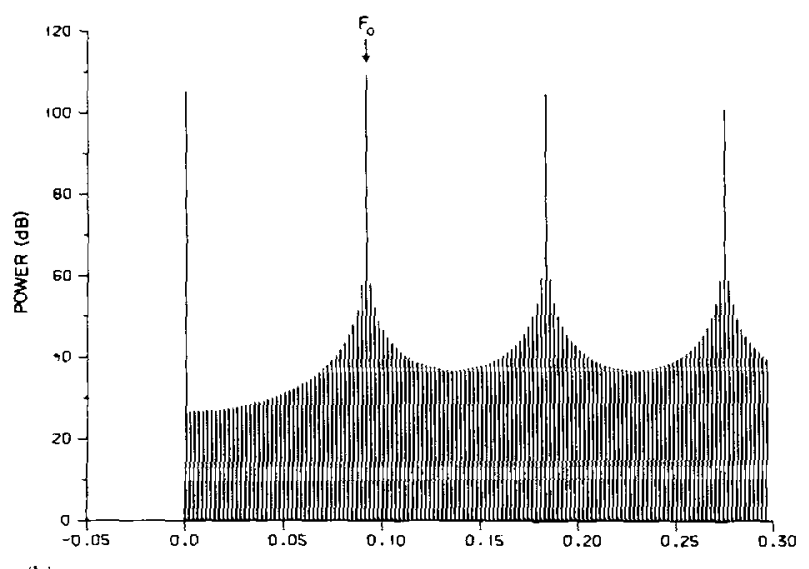

(b)

FREQUENCY - $i / t$

FIG. 4. Dynamics of first Fiske step. Power spectrum of $\phi_{t}$ at left junction end for $\gamma=0.55$ and $\eta=0.75$. (a) Subharmonic branch, (b) major portion of first FS.

branches, the relative ratios of the subharmonic components vary; however, until we reach the juncture point of the branch with the major portion of the FS, motion along the branches is repeatable and reversible. Once the juncture point is reached, however, a simply periodic solution corresponding to the major portion of the FS is maintained, which is also repeatable and reversible. The only way to proceed from the major portion of the FS to the branches is through tickling the solution, which causes a jump to the branch. This may be related to the fact, as is evident from Fig. 1, that the branches have negative differential resistance. When the bias is progressively increased on the lower branch, the junction switches abruptly from the branch to the major portion of the Fs for $\gamma>0.5407$. The same procedure on the upper branch leads to a jump to the third FS for $\gamma>0.5505$. On the major portion of the step, the junction switches to the third FS for $\gamma>0.550$ and to a static zero-voltage state for $\gamma<0.528$. Finally, our results suggest the possible existence of further fine structure of the branches. This is presently being investigated.

We are pleased to thank A. C. Scott for helpful and stimulating discussions. The financial support of the Danish Natural Science Research Council under grant No. 11-3064 and of the European Research Office of the United States Army through contract No. DAJA37-82-C-0057 is gratefully acknowledged.
'S. N. Emé and R. D. Parmentier, J. Appl. Phys. 51, 5025 (1980).

${ }^{2}$ B. Dueholm, O. A. Levring, J. Mygind, N. F. Pedersen, O. H. Soerensen, and M. Cirillo, Phys. Rev. Lett. 46, 1299 (1981).

${ }^{3}$ P. S. Lomdahl, O. H. Soerensen, and P. L. Christiansen, Phys. Rev. B 25, $5737(1982)$.

${ }^{4}$ B. Dueholm, E. Joergensen, O. A. Levring, J. Mygind, N. F. Pedersen, M. R. Samuelsen, O. H. Olsen, and M. Cirillo, Physica B 108, 1303 (1981). ${ }^{5}$ S. N. Erné, A. Ferrigno, and R. D. Parmentier (unpublished).

${ }^{6} \mathrm{M}$. Radparvar and J. E. Nordman, presented at the 1982 Applied Superconductivity Conference.

${ }^{7}$ E. Joergensen, V. P. Koshelets, R. Monaco, J. Mygind, M. R. Samuelsen, and M. Salerno, Phys. Rev. Lett. 49, 1093 (1982).

${ }^{8}$ A. Barone, W. J. Johnson, and R. Vaglio, J. Appl. Phys, 46, 3628 (1975). ${ }^{9}$ N. F. Pedersen (private communication). 